\section{Correcting Scanning Errors in Scanning Probe Microscopes}

Gregor Hsiao, Jezz Leckenby

ThermoMicroscopes, Sunnyvale, CA 94089

Scanning probe microscopes (SPMs) are a family of instruments used for studying the surface properties of materials on a dimensional scale ranging from the atomic to the micrometer level $^{1,2}$. As depicted in Figure 1, all SPMs work by scanning a finely tipped probe in a raster pattern over the sample surface while measuring and mapping some interaction between the probe and the surface as a function of $x-y$ position. The piezoelectric scanners used to provide the scanning motion offer very fine positional control but have certain inherent errors that, uncorrected, can distort images, introduce artifacts, and degrade measurement accuracy. There are two approaches to correcting scanner errors-predictive software corrections that try to anticipate required corrections without actual knowledge of the probe position, and closed-loop feedback corrections (Figure 2) that measure the scanner position at each data point and feed the information back to the scan controller.

Since scan errors can be significant in many common SPM applications, it is important to recognize the conditions under which they are most likely to occur. Here we will look briefly at the most common instances of scanner-induced distortion and at the correction solutions offered by some current generation commercial SPMs. Finally, we'll close with several tests that can be used to evaluate the scan errors and correction capabilities of your own SPM.

\section{Piezoelectric Scanners}

In virtually all SPMs, piezoelectric scanners provide the extremely fine positioning control needed to move the probe tip over the sample. Only piezoelectric scanners offer the small motion increments required for high-resolution SPM. The scanners are made of piezoelectric materials-ceramics that change dimensions in response to an applied voltage as molecular dipoles within the crystalline structure reorient themselves to align with the electric field. They can be designed to move in $x, y$, and $z$ directions. Electromechanical actuators

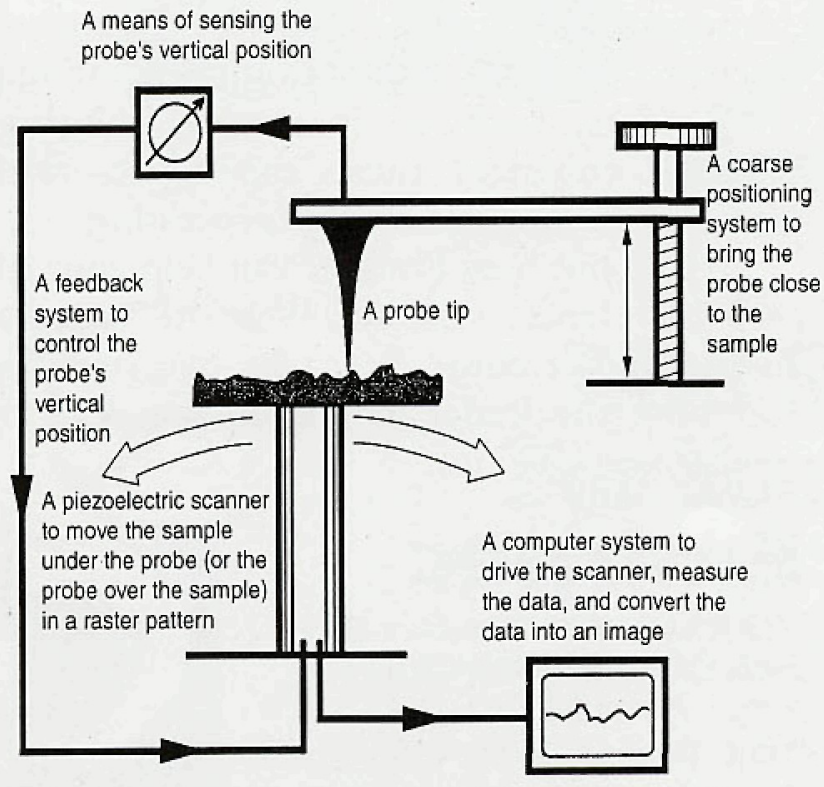

Figure 1: All SPMs contain the components illustrated above. made of these materials consume little power and have few mechanical vibrations.

In the ideal case, the piezo ceramic would deform in a linear manner as the applied voltage increased or decreased. In practice, piezo ceramics do not behave this way. Instead they exhibit intrinsic non-linearity, hysteresis, and creep, as well as extrinsic irregularities that include aging, temperature dependence (the piezoelectric property degrades rapidly above $150^{\circ} \mathrm{C}$ ), and usage effects.

\section{Non-linearity, Hysteresis and Creep}

Scanner non-linearity refers to the fact that scanner extension $\frac{\pi}{2}$ is not exactly proportional to the applied voltage. Typically, the $\stackrel{?}{\equiv}$ extension rate $(\mathrm{dx} / \mathrm{dV})$ is a little higher in the middle of the useful range than at the extremes. Hysteresis introduces another complication into the extension function. Because of hysteresis the ex- $\frac{3}{0}$ tension at most voltages is different if approached from a higher voltage than if approached from a lower voltage. The amount of $\stackrel{5}{c}$ hysteresis depends on the nature and structure of the ceramic, typically hot-pressed, sintered lead zirconium titanate (PZT). Finally, "creep" is a slowly decaying extension that continues after the initial response to a change in voltage. While the initial response occurs in milliseconds, creep can continue for minutes.

\section{Scan Error Distortions}

Image distortions caused by scanning errors can occur when changing scan location, zooming, rotating the scan direction, changing the $z$-piezo extension (as when measuring step heights or the profile of a rough surface), and switching scan rates.

Non-linearity distorts the data-sampling grid in the plane of the sample surface (the $x-y$ direction). In an image of a surface with straight, periodic structures, a diffraction grating for example, non-linearity appears as curvature and non-uniform spacing. On samples without regular repeating features non-linearity may go unnoticed. In the $z$-direction, perpendicular to the sample surface, non-linearity causes errors in feature height measurements made at different total z-piezo extensions.

Scanner hysteresis can be troublesome when trying to return to a previously scanned area. Although the scanner voltages indicate an identical scan location, the new and old images do not

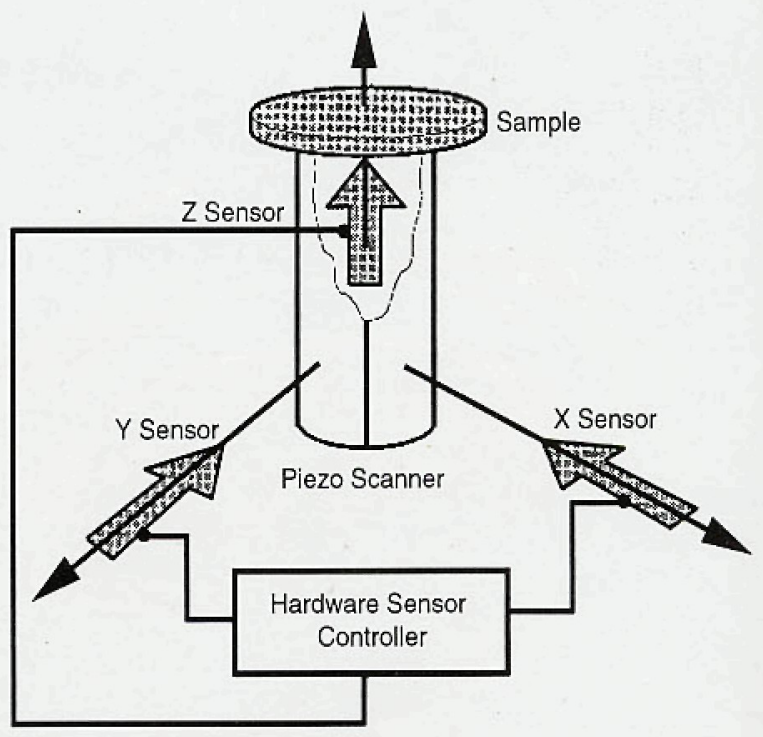

Figure 2: $X, y$ and $z$ position sensing for closed loop feedback scanner control. 
align, and multiple iterations are required to achieve a good match.

$X-y$ creep causes slightly different magnifications in images taken at different scan speeds. Creep can also show up when changing from low to high magnification, as when zooming in for a high-resolution image of an interesting feature located using a wide-field view. The SPM applies an offset voltage to the scanner to move the feature to the center of the new scan. At the new high magnification the targeted feature will either drift into or out of view as the scanner creeps to its final position-a process that can take several minutes.

\section{Software Correction Methods}

\section{Scanning Error Corrections}

Software error correction schemes attempt to predict scanner behavior under frequently used conditions. The corrections may be applied to the scanner during data collection or to the data array after collection is completed. The simplest method applies non-linear drive voltages to the scanner. This approach requires different corrections for each scan range, rotation and scan speed. Post acquisition schemes usually compare the collected data to a known standard and calculate the corrections required to transform the acquired image into a "true" image.

All open-loop software corrections lack direct positional feedback and rely on up-to-date calibration data for accuracy. Although open-loop software-based error correction is generally less expensive to acquire than closed-loop compensation with position sensors, its requirement for careful, periodic calibration adds significantly to operating costs. Even when properly calibrated, software corrections are not always sufficient. Generally, they do not attempt to correct for extrinsic errors such as the aging of the ceramic and the usage patterns of the scanner.

\section{Hardware Correction Methods}

These methods use closed-loop feedback from a sensing device that measures the scanner's actual position. The scan controller uses the feedback signal to drive the scanner to the desired position. Three commonly used methods are:

- Optical Feedback ${ }^{3}$. A light shines through a slit mounted on the scanner onto a two-section photodetector. The photodetector signal is proportional to scanner displacement. Optical feedback

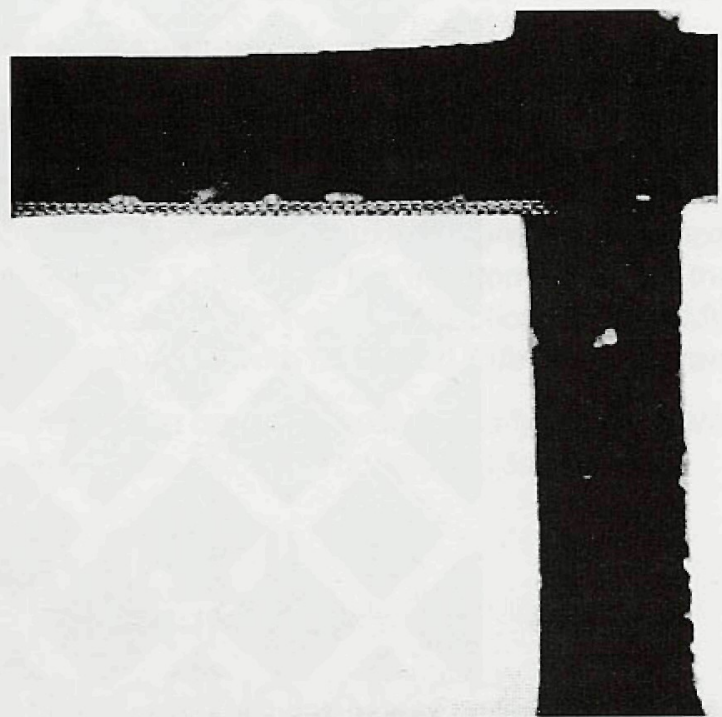

is the fastest technique and permits the highest scan rates.

- Strain Gauge Feedback ${ }^{4}$. Strain gauges measure strain in the $x, y$ and $z$ directions. The strain signal is proportional to displacement.

- Capacitative Feedback. The position detector measures the capacitance between plates mounted on the scanner and at fixed reference positions. The scan controller calculates the scanner's position from the capacitance values. Capacitative feedback is the slowest of the three methods discussed here and can result in prohibitively long image acquisition times.

All of these methods provide real-time correction at each sampling point in the data array. Closed-loop control corrects both intrinsic and extrinsic scanner errors to provide accurate images and measurements under all conditions.

\section{Evaluating Scan Error Distortion on your SPM}

Commercial SPMs use different strategies to mitigate distortion. It is important to know the correction capabilities of your SPM and to recognize situations when scanning errors are likely to distort your images and measurements. Following are some simple tests that can help you determine the efficacy of the scanning error corrections on your SPM.

\section{- Zoom}

This test detects non-linearity, hysteresis and creep. An appropriate sample is a $10 \mu \mathrm{m} x-y$ diffraction grating. The imaging mode can be contact or non-contact AFM with an appropriate tip.

1. Image an area of $100 \times 100 \mu \mathrm{m}$ at $1.0 \mathrm{~Hz}$ scan speed.

2. Image an area of $10 \times 10 \mu \mathrm{m}$, offset to the lower left of the large image $(x=-37.5, y=-37.5)$ at $0.75 \mathrm{~Hz}$.

3. Image an area of $10 \times 10 \mu \mathrm{m}$, offset to the upper right of the large image $(x=+37.5, y=+37.5)$ at $1.5 \mathrm{~Hz}$.

4. Image an area of $10 \times 10 \mu \mathrm{m}$, offset to the lower left of the large image $(x=-37.5, y=-37.5)$ at $1.0 \mathrm{~Hz}$. This is the same area as in step 2.

5. Repeat Step 1 two times and compare images.

Performing this test without delay between steps will make any hysteresis-induced distortion quite apparent. The lines of the grating will be curved instead of straight and the degree of curvature will depend on the scan rate. Finally, the areas displayed in Step 1

\section{Continued on following page}
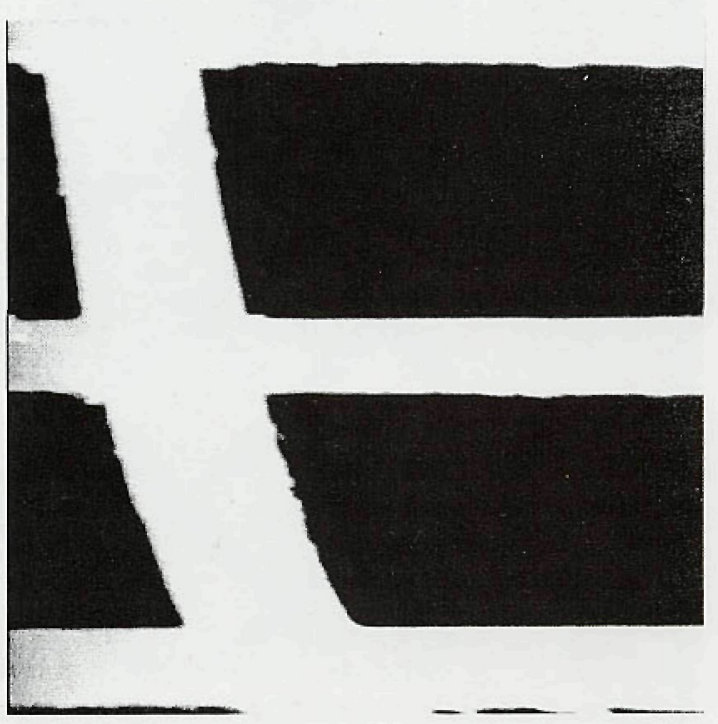

Figure 3: The sample in both images above is a symmetrical diffraction grating with square features repeating every 10 micrometers. The left hand image was taken using closed loop feedback to correct for scanner errors. The image on the right was taken using only software corrections. 


\section{Correcting Scanning Errors in Scanning Probe Microscopes \\ Continued from preceding page.}

and Step 4 will not match.

Figures $3 a$ (with closed loop feedback) and $3 b$ (without closed loop feedback) compare the results of this test using two different microscopes.

\section{- Scan Rotation}

Often upon imaging you find that the sample is not aligned correctly. The sample can be aligned by physically rotating it, which may involve some trial and error, or, on most SPMs, it can be aligned by rotating the scan direction. This can introduce scanning errors. Perform this test using a $10-\mu \mathrm{m} x-y$ diffraction grating as in the Zoom test.

1. Image an area of $50 \times 50 \mu \mathrm{m}$ at $1.0 \mathrm{~Hz}$ scan speed without any position offsets.

2. Image the same area with a scan rotation of $45^{\circ}$. Don't change anything else.

3. Image the same area with a scan rotation of $340^{\circ}$. Don't change anything else.

8. Analyze the unprocessed data. You may notice a slight waviness to the grid lines. Measure the distance between the grid lines by drawing a line across the image parallel to the lines. Do this again for a second line perpendicular to the first line. If the distances between individual grid lines vary from the first grid to the last, there is distortion.

Figures $4 \mathrm{a}$ (with closed loop correction) and $4 \mathrm{~b}$ (without closed loop correction) compare the results of this test on two different microscopes. In figure $4 a$, segments $a, b$ and $c$ measured $9.97,9.97$, and 9.97 micrometers in length. The straight line drawn perpendicular to the measured segments demonstrates the lack of distortion in the image. In figure $4 \mathrm{~b}$, segments $a, b$, and c measured $8.98,9.37$ and 9.57 respectively, a relative range of more than $5 \%$. The distortion in the image becomes apparent in comparison to the perpendicular line.

\section{- Height and Roughness Measurement}

This test measures inaccuracy caused by non-linearity and hysteresis in the z-direction. An appropriate sample is a z- height or step standard with $180 \mathrm{~nm}$ steps. Use contact AFM mode with an appropriate tip.

1. Image an area of 3-4 $\mu \mathrm{m}$ that includes the steps. The $z$-piezo extension should be about $50 \%$.

2. Offset the scanning head $z$ motor so that the $z$-piezo is $15 \%$ extended. Image the same area again with no other changes.

3. Offset the scanning head $z$ motor so that the $z$-piezo is $85 \%$ extended. Image the same area again with no other changes.

4. Offset the scanning head $z$ motor so that the $z$-piezo is $40 \%$ extended. Image the same area again with no other changes.

5. Offset the scanning head $z$ motor so that the $z$-piezo voltage is $60 \%$ extended. Image the same area again with no other changes.

Measure the step height for each of the images obtained. The amount of variation in the step height (for example, the standard deviation) is a measure of the inaccuracy caused by $z$ nonlinearity.

\section{Conclusion}

SPM scanning errors can introduce significant image distortion and measurement error. Hardware corrections using closed loop feedback from position sensors are superior to predictive software corrections. These errors are not always easy to detect, especially on irregular samples. It is important to evaluate the scan error correction capability of your SPM. Most manufacturers offer closed loop feedback corrections, although sometimes at significant additional cost. Carefully consider the cost of accurate corrections versus the potential cost of inaccurate data.

\section{References}

1) R. Howland and L. Benatar, "A Practical Guide to Scanning Probe Microscopy," Park Scientific Instruments (1997). Available from ThermoMicroscopes, Sunnyvale, CA 94089.

2) "Artifacts in SPM," TopoMetrix (1993). Available from ThermoMicroscopes, Santa Clara, CA 95054.

3) ScanMaster ${ }^{T M}$ US Patent $5,376,790$, ThermoMicroscopes, Sunnyvale, CA.

4) TrueMetrix ${ }^{T M}$ US Patent $5,469,734$, ThermoMicroscopes, Sunnyvale, CA.
4A

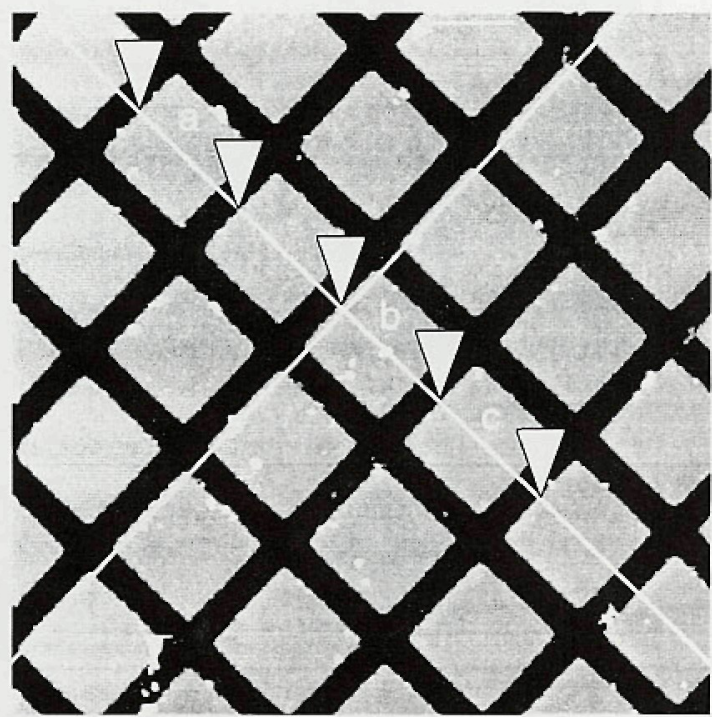

4B

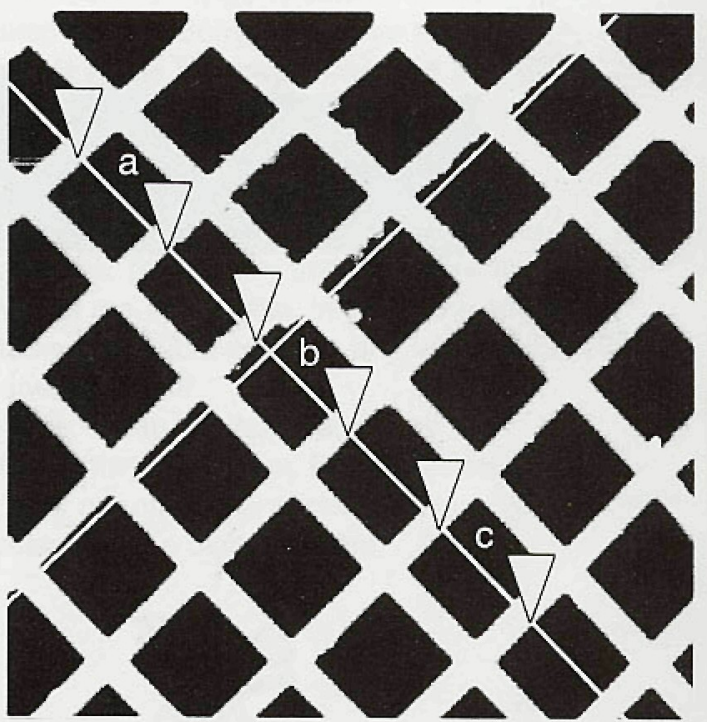

Figure 4: In both images above, the scan direction has been rotated. In the lefthand image, acquired using closed loop scan correction, segments $a, b$, and $c$ each measure 9.97 micrometers in length. In the righthand image, acquired using software scan corrections, the segments measured $8.98,9.37$, and 9.57 micrometers, respectively. The straight lines drawn perpendicular to the segments in each image make the curvature introduced into the right hand image by scanning errors very apparent. 


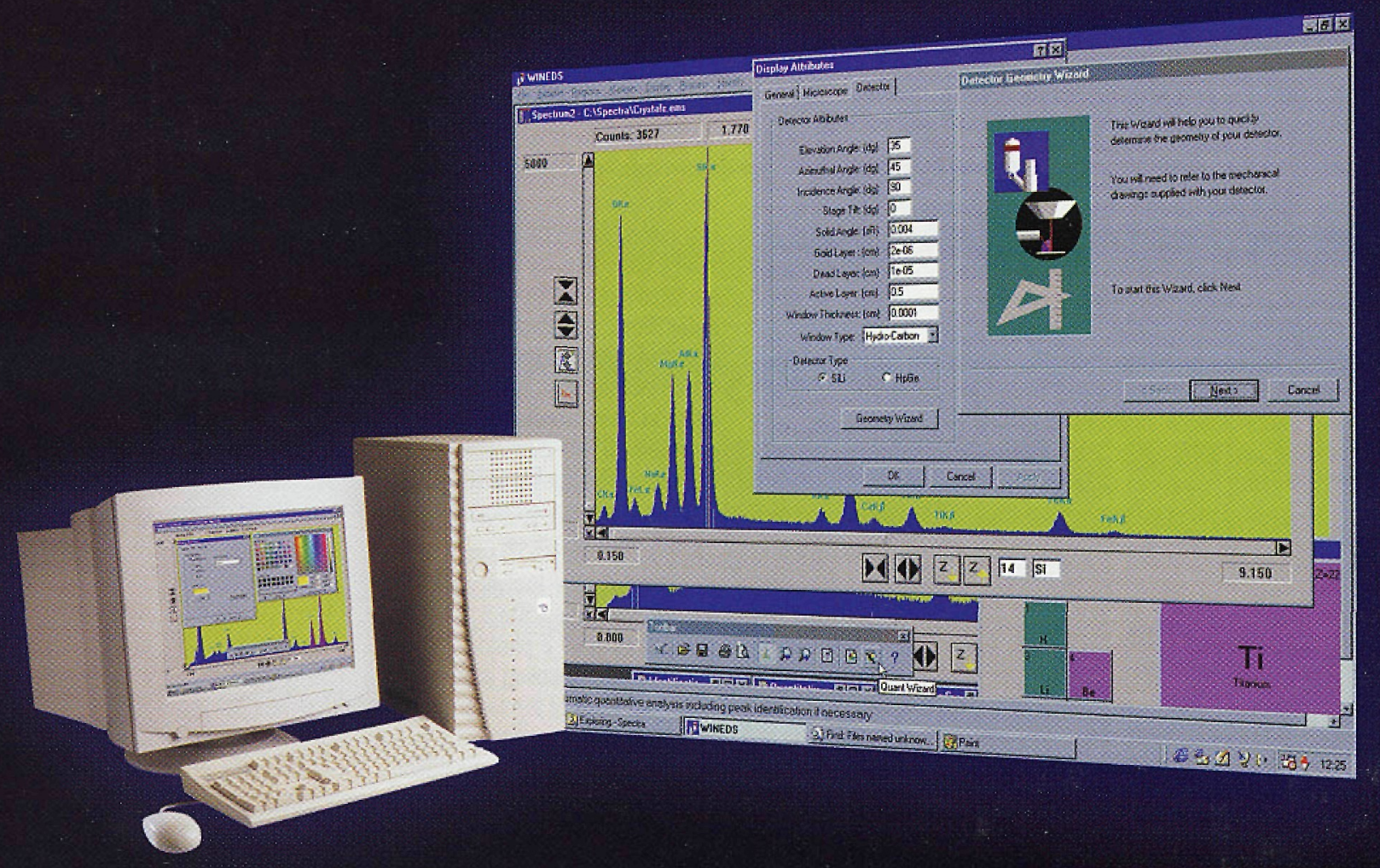

\section{When looking at things closely,}

\section{WIN $=D S^{\odot}$ is easier to use and better value than any other Microanalysis system.}

\section{THE SOFTWARE}

The clever thing about WINEDS ${ }^{\oplus}$ is it behaves just like all your other Windows'm applications. In fact you probably already know how to use it because it is fully compliant with Windows'm 95/98/2000 and NT 4.0 interfaces. It features:

- New Quant Wizard one step analysis

- New Geometry Wizard interactively determines detector geometry

- Smart validation of analysis parameters

- New "Tool Tips" and "Hint Text" captions

- Multiple fully re-sizeable Spectrum Windows

- Easy report generation

- Windows ${ }^{\mathrm{TM}} 2000$ ready / Y $2 \mathrm{~K}$ compliant

\section{THE COMPLETE SYSTEM}

We offer a complete range of high performance microanalysis systems including: PC, detector electronics, detector and digital imaging.

Our products are also available as an ideal upgrade package for older microanalysis systems.

\section{THE VERDIGT}

WINEDS ${ }^{\circledR}$-the world's most user friendly microanalysis system that will fit your budget.

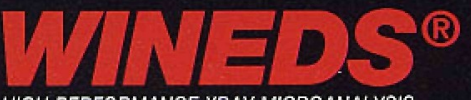

HIGH PERFORMANCE XRAY MICROANALYSIS
Thomson Scientific Instruments Pty Ltd $A C N$ O 05720945

216 DRUMMOND ST, CARLTON 3053 , VICTORIA AUSTRALIA TELEPHONE (03) 96632738 (03) 96632742 FAX (03) 96633680

INTERNATIONAL FAX 61396633680

E-MAIL: tsi@mira.net

WEB PAGE: http://www. werple.net.au/ tsi/
USA

Telephone 6087982005

Fax 6087981675

Email doug_tnasqüclassic.msn.com 